\title{
UM ESTUDO SOBRE DEPRESSÃO NA TERCEIRA IDADE
}

\section{A STUDY ON DEPRESSION IN THE ELDERLY}

Claudia Moreira de Lima'; Nêmora da Silva Barros²; Bárbara Maria Antunes Barroso3; Anna Carolina Daltro Pereira4; Ana Paula Vasconcellos da Silva ${ }^{5}$.

${ }^{1}$ Enfermeira. Mestranda do Programa Ambiente e Saúde da Universidade de CuiabáUNIC.. cml claudiamoreira@hotmail.com.

${ }^{2}$ Enfermeira. Docente Assistente da Universidade do Estado de Mato Grosso

(UNEMAT), Campus Universitário de Diamantino.nemorabfaria@gmail.com

${ }^{3}$ Enfermeira. Universidade do Estado de Mato Grosso (UNEMAT), Campus Universitário de Diamantino. Docente da Secretaria de Estado de Ciência e Tecnologia de Mato Grosso. barbarabarroso@hotmail.com.

${ }^{4}$ Enfermeira Mestre em Saúde Coletiva pela Universidade Federal de Mato Grosso (UFMT). Docente Assistente da Universidade do Estado de Mato Grosso (UNEMAT), Campus Universitário de Diamantino. Annacdp25@gmail.com.

${ }^{5}$ Enfermeira. a p vasconcellos@hotmail.com.

\section{Endereço para correspondência:}

Claudia Moreira de Lima . Rua: Manoel Jose de Arruda ํㅜ 3.100 - Bairro: Jardim Europa - Cuiabá - MT, 78065-900. cml claudiamoreira@hotmail.com. 


\section{RESUMO}

A população brasileira está envelhecendo consideravelmente, no entanto aliado a este crescimento da idade populacional surgem problemas econômicos, físicos e mentais, sendo as doenças mentais com grande destaque, sendo a depressão o transtorno mental mais presente entre os idosos, uma vez que o idoso com o avançar da idade perde sua autonomia ficando menos ativo, levando a perda da qualidade de vida podendo ocasionar um isolamento social e consequentemente a depressão se instala. Diante dessa realidade buscou-se com o estudo elencar a problematização entre idoso e depressão, sendo realizado para isso uma pesquisa de natureza básica de objetivo descritivo, com abordagem qualitativa a partir de ensaio bibliográfico, com busca em artigos na base de dados da Biblioteca Virtual em Saúde (BVS), com período de busca compreendido entre 2010 a 2016. Os resultados mostram a necessidade de investir em estudos relacionados as especificidades do idoso, onde a temática além de ser importante é atual, entretanto seus achados ainda são escassos.

Descritores: Envelhecimento, Depressão, Sistemas de Saúde.

\section{ABSTRACT}

The Brazilian population is aging considerably, however, coupled with this growth of the population age, economic, physical and mental problems emerge, with mental illnesses being the most prominent, with depression being the most present mental disorder among the elderly, since the elderly with the advancement of age loses its autonomy becoming less active, leading to loss of quality of life that can lead to social isolation and consequently depression settles. In view of this reality, the study was aimed at elucidating the problematization between the elderly and depression. A basic objective research was carried out with a qualitative approach based on a bibliographical essay, with a search of articles in the Virtual Library database (VHL), with a search period between 2010 and 2016. The results show the need to invest in studies related to the specificities of the elderly, where the subject matter besides being important is current, however their findings are still scarce.

Descriptors: Aging, Depression, Health Systems. 


\title{
INTRODUÇÃO
}

Envelhecer é um processo natural que caracteriza uma etapa da vida do homem sendo idoso segundo o Estatuto do Idoso Lei 10.741/03, todo indivíduo com idade igual ou superior a 60 anos, contudo, é valido o que nos traz Netto ${ }^{1}$ ( . 10) onde afirma que "... não há uma consciência clara de que através de características físicas, psicológicas, sociais, culturais e espirituais possa ser anunciado o início da velhice" ${ }^{2,3}$.

A população brasileira vem envelhecendo consideravelmente nos últimos anos justificando esse fenômeno aos avanços da saúde e da tecnologia que concorrem para favorecer um envelhecimento mais saudável e com melhor qualidade de vida, o que levou a uma perspectiva de aumento para 32 milhões de idosos com 60 anos ou mais em 2025 4,5,6,7. O Instituto Brasileiro de Geografia e Estatística, IBGE evidencia essa realidade, ao relatar que:

\begin{abstract}
O segmento populacional que mais aumenta na população brasileira é o de idosos, com taxas de crescimento de mais de $4 \%$ ao ano no período de 2012 a 2022. A população com 60 anos ou mais de idade passa de 14,2 milhões, em 2000, para 19,6 milhões, em 2010, devendo atingir 41,5 milhões, em 2030, e 73,5 milhões, em 2060. Espera-se, para os próximos 10 anos, um incremento médio de mais de 1,0 milhão de idosos anualmente. Essa situação de envelhecimento populacional é consequência, primeiramente, da rápida e contínua queda da fecundidade no país, além de ser também influenciada pela queda da mortalidade em todas as idades (IBGE ${ }^{8}$, p. 146).
\end{abstract}

Conceitualmente envelhecer é um processo natural, progressivo e universal que vem com inúmeras alterações no organismo, onde se manifesta de forma variável, envolvendo dimensões tanto biológicas, cronológicas e social, entretanto com o envelhecimento advém problemas econômicos, físicos e mentais 9,5.

$O$ envelhecimento pode ser normal (senescência) o qual proporciona aos indivíduos conviverem de forma harmoniosa com as limitações impostas pela idade, ou pode ser um período ou marcado por condições que prejudicam a funcionalidade deste indivíduo nas suas atividades da vida diária (senilidade), ou seja o envelhecimento se dá de forma anormal ou patológica, impondo um efeito negativo das doenças causando em sua maioria uma incapacidade progressiva a vida ${ }^{10,11}$.

Destarte ao aludido é mister que o número de indivíduos com demência é estimado em 35,6 milhões, sendo esperado em 2030 um total de 65,7 milhões ${ }^{12}$. 
Desta feita a Organização Mundial da Saúde (OMS) aponta como as principais doenças que afetam os idosos as doenças cardiovasculares, hipertensão, derrame, diabete, doença pulmonar obstrutiva crônica (DPOC), doenças musculoesqueléticas, com grande destaque para as doenças mentais ${ }^{11}$. Sendo doenças mentais condições de anormalidades ou comprometimento que afetam a ordem psicológicas, mental ou cognitiva, podendo ser explicadas pela genética, problemas bioquímicos e o estilo de vida do indivíduo. No geral umas das doenças mentais mais comum e conhecida é a depressão ${ }^{13}$.

A depressão é caracterizada por um distúrbio multifatorial que atinge a área afetiva sendo ocasionada por aspectos de ordem biológica, psicológica e social, tendo como principais sintomas o humor deprimido e a perda de interesse ou prazer na realização das atividades. Sendo dentre as doenças mentais a de maior prevalência em nível mundial, estimando que em 2020 ocupara o segundo lugar das doenças incapacitantes acometidas pelo indivíduo. Na população idosa, essa é uma doença comum, recorrente e frequentemente subdiagnosticada e subtratada, tendo maiores índices de ocorrências nas mulheres idosas ou idosos com idades mais avançada que acumulam morbidades e/ou fragilidades que comprometem a qualidade de vida deste indivíduo 14,15,16,17,18,19.

Apesar desta realidade hodiernamente o diagnóstico de depressão em idosos é difícil de se concluir e mesmo quando identificado estes muitas vezes se recusam ou demonstram resistência em aceitar que está com alguma doença mental e que esta doença é a depressão, uma vez que a mesma tem seu diagnóstico por investigação e não por um exame especifico. Sendo assim necessário investir em estudos nas questões relacionadas as especificidades da saúde do idoso ${ }^{18}$.

Frente a essa problemática e à relevância do tema exposto, justifica-se a necessidade de buscar evidencias que apontem para o desvendar da depressão e idoso, no intuito de condensar e disseminar o conhecimento produzido a respeito e auxiliar na melhoria da atenção ofertada a esses indivíduos. Diante desses dados o objetivo deste estudo é verificar a produção científica nacional acerca das relações entre depressão, idosos e enfermagem.

\section{MÉTODOS}


A pesquisa foi desenvolvida utilizando o método de revisão bibliográfica, pois a revisão é a base que sustenta qualquer pesquisa cientifica, sendo indispensável para delimitação do problema em um projeto de pesquisa, e para obter uma ideia precisa sobre o estado atual do conhecimento sobre o tema, sua lacunas e contribuição da investigação para desenvolvimento do conhecimento ${ }^{20}$.

Para o desenvolvimento desta revisão, foram percorridas as seguintes etapas: definição do tema e formulação da questão norteadora; escolha das bases de dados eletrônicos utilizadas na pesquisa; estabelecimento dos critérios de inclusão e exclusão; definição dos descritores; pré-seleção dos artigos; avaliação dos estudos pré-selecionados e seleção dos estudos incluídos na revisão; interpretação dos resultados e apresentação dos achados.

Compreendendo a problematização apresentada, foi elaborada a questão norteadora acerca da situação: Quais a evidência da produção cientifica brasileira nos últimos 07 anos, acerca da temática processo de envelhecimento e depressão senil? A busca do objeto de estudo foi realizada através da Biblioteca Virtual em Saúde (BVS), visando atender a recomendação da literatura que se busquem diferentes fontes de dados.

O levantamento bibliográfico, ocorreu no mês de fevereiro de 2017, na respectiva base de dados da Biblioteca Virtual em Saúde (BVS), utilizando os descritores: "Idoso, Depressão e Enfermagem". Desta forma, a estratégia de pesquisa constituiu-se do booleano conector: "AND", e as buscas realizadas foram: "Envelhecimento AND depressão", "Envelhecimento AND Serviços de Saúde", "Depressão AND Serviços de Saúde", "Envelhecimento AND depressão AND Serviços de Saúde".

Para seleção dos artigos foram estabelecidos como critérios de inclusão: textos completos, idioma (português), ano de publicação (2010 a 2016) e que correspondiam à temática abordada. Os critérios de exclusão foram: dissertações, teses, monografias, livros e artigos de periódicos não científicos; artigos não disponíveis na íntegra; artigos que não se enquadravam no período de publicação determinado e artigos que não contemplavam a temática abordada no estudo.

Foram encontrados 61.762 trabalhos, que após o crivo de acordo com os critérios de inclusão e exclusão, foram selecionadas 74 publicações, que foram 
submetidas a uma leitura exploratória, a qual se caracteriza por ser uma leitura corrida, objetivando evidenciar se o texto atendia ao objetivo proposto, este momento resultou na exclusão de 45 (quarenta e cinco) trabalhos. Desse montante 15 (quinze) artigos foram excluídos por não corresponderem a temática, logo desse total 7 (sete) encontravam-se repetidos na página de dados e 1 artigo permaneceu em outro idioma após a filtragem da temática. Assim obtivemos como amostra final do estudo, 6 (seis) artigos.

\section{RESULTADOS E DISCUSSÃO}

Após a coleta de dados todas as informações foram catalogadas através dos indicadores, codificadas e analisadas, sendo os estudos identificados por códigos (art.1, art.2, assim por diante). Os dados foram analisados de modo descritivo agrupados por temas pertinentes ao assunto estudado.

Tabela 01: Estudo categorizados de acordo com título e ano de publicação do estudo. Diamantino-MT, 2017.

\begin{tabular}{l|l|l}
\hline SIGLA & TITULO DO ARTIGO ANO DE & PUBLICAÇÃO \\
\hline Art.01 & $\begin{array}{l}\text { Capacidade funcional declínio cognitivo e depressão } \\
\text { em idosos institucionalizados: possibilidade de } \\
\text { relações e correlações }\end{array}$ & 2012 \\
\hline Art.02 & $\begin{array}{l}\text { Fatores associados à síndrome metabólica em idosos } \\
\text { do interior do Nordeste brasileiro }\end{array}$ & 2016 \\
\hline Art.03 & $\begin{array}{l}\text { Prevenção e promoção da saúde mental no } \\
\text { envelhecimento: conceitos e intervenções }\end{array}$ & 2014 \\
\hline Art.04 & $\begin{array}{l}\text { Qualidade de vida, sintomas depressivos e } \\
\text { religiosidade em idosos: um estudo transversal }\end{array}$ & 2014 \\
\hline Art.05 & $\begin{array}{l}\text { Reflexos do tempo: uma reflexão sobre o } \\
\text { envelhecimento nos dias de hoje }\end{array}$ & 2015 \\
\hline Art.06 & $\begin{array}{l}\text { Envelhecimento populacional e doenças crônica / } \\
\text { reflexão sobre os desafios para o sistema de saúde } \\
\text { publica }\end{array}$ & 2015 \\
\hline
\end{tabular}


Fonte: Do pesquisador.

Diante do estudo realizado observa-se na tabela 1 que não houve constância nas publicações encontradas apresentando oscilação em relação ao ano de publicação. Os anos de 2010, 2011 e 2013 não houveram publicações sendo que nos anos seguintes (2012 a 2016) as publicações variaram entre 1 a 2 em cada ano.

Buscando uma melhor compreensão frente à temática estudada nessa pesquisa, identificamos a ideia central dos estudos da amostra, demonstrados na tabela 2.

Tabela 2- Ideias centrais dos estudos da amostra. Diamantino-MT, 2017.

\begin{tabular}{|c|c|}
\hline $\begin{array}{l}\text { CÓDIGO DO } \\
\text { ARTIGO }\end{array}$ & IDEIA CENTRAL \\
\hline Art.01 & Analisa a relação do declínio e a depressão. \\
\hline Art.02 & Associação das doenças que levam a depressão. \\
\hline Art.03 & $\begin{array}{l}\text { Descreve a percepção do envelhecimento na } \\
\text { atualidade. }\end{array}$ \\
\hline Art.04 & Investiga os conceitos de depressão. \\
\hline Ar.05 & $\begin{array}{lcccc}\text { Analise da maneira } & \text { como } & \text { lidamos } & \text { com } \\
\text { envelhecimento } & & & & \\
\end{array}$ \\
\hline Art.06 & $\begin{array}{l}\text { Analisa o sistema de saúde, diante das respectivas } \\
\text { doenças da população idosa }\end{array}$ \\
\hline
\end{tabular}

Fonte: Do pesquisador.

A tabela 3 apresenta a produção científica sobre idoso e depressão segundo categoria temática. Essas categorias emergiram após a leitura seletiva, elegendo e avaliando a contribuição de cada texto e seus principias tópicos, o que foi essencial para apresentação dos achados.

Tabela 3: Produção científica segundo categoria temática dos estudos. DiamantinoMT, 2017. 


\begin{tabular}{l|l}
\hline CATEGORIAS ENCONTRADAS & CÓDIGO DOS ESTUDOS \\
\hline $\begin{array}{l}\text { Relação cognitivas e metabólicas da } \\
\text { depressão }\end{array}$ & Art. 01 e 02 \\
\hline Fatores que geram a depressão & Art -.03 \\
\hline Entendendo o envelhecimento & Art. 04 e 05 \\
\hline Sistema de saúde e o idoso & Art. 06
\end{tabular}

Fonte: Do pesquisador.

Todo indivíduo passa por etapas e mudanças na sua vida, mudanças elas físicas e sociais, sendo o envelhecimento algo natural de todo ser humano, destarte o envelhecimento pode ser visto como um processo universal, progressivo e dinâmico que se inicia na concepção e finda com a morte, sendo necessário ações que promovam e previnam o surgimento de quadros clínicos de agravo a saúde do indivíduo em todas as suas fases da vida, em especial no envelhecimento, garantindo uma qualidade de vida neste período ${ }^{21,22}$.

O envelhecimento está na maioria das vezes associado a doenças crônicas, que veem surgindo com passar dos anos, nos conduzindo a pensar que é um ciclo do processo envelhecer, sendo uma das doenças mais corriqueiras hodiernamente de acometer o indivíduo idoso a depressão, sendo mister identificar seus sinais e como ambas (velhice e depressão) se manifestam no cotidiano da pessoa idosa $^{23,24}$.

A depressão é tida como um transtorno de humor/afetivo que surgiu no século 18 sobre o contexto psiquiátrico europeu, com características que predominam a perturbação de humor, alterando seu estado emocional ${ }^{25,26}$.

Diante do decorrer dos tempos, foram muitas ideias para avaliar o termo depressão, que ainda hoje vem sido contextualizado de muitas formas, correlacionando diversos fatores, dais quais muda a forma de diagnosticar a depressão, onde esta não é uma doença rotulada, onde dormimos e acordamos com ela, mas é sucinta e surgi gradativamente e silenciosamente, agredindo o indivíduo de forma que passe despercebido no seu cotidiano, podendo assim se 
tornar alvo fácil para se alienar a alguma outra doença e desencadear um quadro grave a saúde podendo resultar como consequência até a morte ${ }^{27,28}$.

É mister observar o comportamento do idoso, compreender as mudanças no corpo e mente, acerca de entender/identificar sinais depressivos. Sendo depressão caracterizada como um transtorno frequente que acaba incapacitando o indivíduo em variados graus a depender do fator acometimento, sendo na terceira idade mais agressivo $^{29}$.

Estudos apontam que durante o processo de envelhecimento o metabolismo e raciocino logico do indivíduo se tornam mais lento até sua parada total, este dado sugere que conforme os avanços dos anos tanto o raciocino quanto o metabolismo desaceleram dando espaço para possíveis patologias, entre elas a depressão nesta fase da vida, uma vez que tanto a síndrome metabólica é uma condição patológica e heterogênica, sendo considerada um fator desencadeante para desenvolver a depressão neste grupo ${ }^{30}$.

Corroboram com estes dados Reuter-Lorenz \& Park ${ }^{31}$, onde afirmam em seu estudo que propriedades do envelhecimento e declínio cognitivo acometem a capacidade funcional do idoso o deixando vulnerável e muitas das vezes excluindo sua vida social, com perda de autonomia e independência, uma vez que suas ideias e funcionamentos físicos se encontram limitados. Diante ao aludido se torna essencial compreender como o raciocínio cognitivo e o metabolismo atuam na vida do idoso 32 .

Visando compreender o processo de envelhecimento e qualidade de vida nesta fase é preciso manter e promover a autonomia e independência dos idosos, a cerca de realizações de ações voltadas para o indivíduo e coletivo se baseando nas diretrizes e princípios do Sistema Único de Saúde - SUS ${ }^{11,33}$.

Desta feita é preciso compreender se o sistema corresponde às respectivas doenças do idoso e o preparo da equipe responsável para seu atendimento. Elencando quais as maiores dificuldades encontrada para dar suporte ao idoso, questionando em que aspectos devem-se melhorar a saúde para este grupo. Procurando por fatores que busquem melhorar cada vez mais a rede de atenção integral para o idoso ${ }^{34}$. 
Somando a compreensão do sistema é mister a atuação de uma equipe de saúde preparada para atender as necessidades da população idoso, uma vez que o Brasil vem envelhecendo gradativamente e se tornando cada dia mais um desafio para assisti-lo e prestar cuidado humanizado. A equipe precisa estar preparada para atender as necessidades deste público, com capacitação técnico cientifica desta fase da vida sendo capaz de cuidar, sanar dúvidas e orientar o indivíduo idoso contribuindo para uma melhora em seu quadro clinico e consequentemente uma qualidade de vida aceitável ${ }^{33}$.

\section{CONCLUSÃO}

O Brasil está em um franco processo de envelhecimento, e neste estudo foram encontrados diversos pontos na relação do envelhecimento e a depressão. Diante ao aludido, notasse que o envelhecimento vem causando um impacto na reflexão acerca do envelhecer, questionando se estamos preparados para lidar com essa geração de idoso que traz uma sobrecarga passada, de um indivíduo que já foi jovem, mas que hoje se torna velho e seus ideais e maneiras de vida se camuflam diante do mundo com ideias diferenciadas da sua época. Nos fazendo abrir paradigmas a respeito das políticas públicas de saúde, nos levando a analisar acerca do despreparo para lidarmos com o idoso e suas manifestações patológicas.

Em suma os resultados deste estudo nos permitem enfatizar na necessidade de uma fonte de pesquisa que aborda esta temática, inserindo na categoria da saúde aberturas para assistir o idoso e suas peculiaridades, dando voz a esses, em troca de fazer uma busca melhorada do quadro do idoso, tentado compreende-lo entender seus sinais e sintomas que podem vir a desenvolver o quadro depressivo.

\section{REFERÊNCIAS}

1. Papaléo Netto $M$. Estudo da velhice no século $X X$ : histórico, definição do campo e termos básicos. In E. Freitas, L. Py, A. L. Neri, F. A. X. Cançado, M. L. Gorzoni \& S. M. Rocha. Tratado de geriatria e gerontologia. Rio de Janeiro: Guanabara Koogan; 2002. p.2-12. 
2. Camarano AA. Envelhecimento da população brasileira: uma contribuição demográfica. IPEA> Instituto de Pesquisa Econômica Aplicada. 2002: 31.

3. Jacob, L. Envelhecimento da população. In. Jacob, L., Santos, E., Pocinho, R. \& Fernandes, H. (Eds.). Envelhecimento e Economia Social: Perspetivas Atuais. Viseu: Psicossoma; 2013. p.43-49.

4. ANDRADE HÁ dos S, Silva SK da, Santos MIP de O. Aids em idosos: vivencias dos doentes. Escola Anna Nery. 2010;14(4):p.712-9.

5. KUCHEMANN BA. Envelhecimento populacional, cuidado e cidadania: velhos dilemas e novos desafios. Soc. estado., Brasília. 2012. p. 165-180.

6. Instituto Brasileiro de Geografia e Estatística [homepage na internet]. Pesquisa Nacional por Amostra de Domicílios. 2012. [acesso em 23 fev. 2017]. Disponível em: http://www.ibge.gov.br/home/presidencia/noticias/imprensa/ppts/0000001013 5709212012572220530659.pdf.

7. Schimidt TCGS, Silva MJP. Percepção e compreensão de profissionais e graduandos de saúde sobre o idoso e o envelhecimento humano. Revista Escola de Enfermagem da USP. São Paulo, SP. 2012. 46(3), p. 612-617.

8. Instituto Brasileiro de Geografia e Estatística [homepage na internet]. Mudanças demográficas no Brasil no início do século XXI - subsídios para as projeções das populações. 2015. [acesso em 23 fev. 2017]. Disponível em: http://biblioteca.ibge.gov.br/visualizacao/livros/liv93322.pdf.

9. Camacho ACLF, Coelho MJ. Políticas públicas para a saúde do idoso: revisão sistemática. Brasília, DF: Revista Brasileira de Enfermagem. 2010. 63(2), 279-284.

10. PASCHOAL SMP. Epidemiologia do envelhecimento. In: PAPALÉO NETTO, M. Gerontologia: a velhice e o envelhecimento em visão globalizada. São Paulo: Atheneu, 2005. p.26-43.

11. Instituto Brasileiro de Geografia e Estatística [homepage na internet]. Instituto Brasileiro de Geografia e Estatística sobre a condição de saúde dos idosos. 2006. [acesso em 23 fev. 2017]. Disponível em: https://biblioteca.ibge.gov.br/visualizacao/livros/liv42597.pdf. 
12. Organização das Nações Unidas. Envelhecimento no século XXI: celebração e desafio. Nova York, EUA: UNFPA. 2012.

13. Soler C. Um mais de melancolia. In: ALMEIDA, CP, MOURA JM (Orgs). A dor de existir e suas formas clinicas: tristeza, depressão, melancolia. Rio de Janeiro: Contracapa Livraria, 1997, p.166-187.

14. Medeiros JML. Depressão no idoso. [Dissertação] [Porto] Faculdade de Medicina, Universidade do Porto. 2010. 31 p.

15. Carreira L. Prevalência de depressão em idosos institucionalizados. Revista Enfermagem UERJ, Rio de Janeiro. 2011; p. 268-273.

16. Alexopoulos GS. Depression in elderly. Lancet. DOI: https://doi.org/10.1016/S0140-6736(05)67091-2.

17. Luppa M, Sikorski C, Luck T, Ehreke L, Konnopka A, Wiese B, et al. Age-and gender-specific prevalence of depression in latest-life - Systematic review and meta-analysis. J Affect Disord. DOI: 10.1016/j.jad.2010.11.033.

18. Santos LM, Cortina I. Fatores que contribuem para a depressão no idoso. Revista de Enfermagem UNISA, Santo Amaro.2011; p.112-116.

19. González LAM. Vivencia de los cuidadores familiares de adultos mayores que sufren depresión. Revista da Escola de Enfermagem da USP, São Paulo. 2010; p. 32-39.

20. Marconi MA, Lakatos EM. Fundamentos de metodologia científica. 7.ed. São Paulo: Atlas, 2010; 310p.

21. LEANDRO-FRANCA C, GIARDINI MURTA S. Prevenção e promoção da saúde mental no envelhecimento: conceitos e intervenções. Psicol. cienc. prof. DOI http://dx.doi.org/10.1590/1982-3703001152013.

22. PAPALÉO NETTO, Matheus; PONTE, José Ribeiro. Envelhecimento: desafio na transição do século. In: PAPALÉO NETTO, Matheus. Gerontologia - A velhice e o envelhecimento em visão globalizada. 2.ed. São Paulo: Atheneu, 2002; p. 3-12.

23. Rosa CM, Veras L, Assunção A. Reflexos do tempo: uma reflexão sobre o envelhecimento nos dias de hoje. Estud. pesqui. psicol. 2015; p. 1027-1044.

24. Martins, RML, Santos ACA. Ser idoso hoje. Millenium - Viseu, 2008; p. 69-76 
25. Ballone G. Depressão no Idoso -in. PsiqWeb [Internet]. 2015. Disponível em www.psiquiweb.net.

26. Teixeira MAR, Hashimoto F. Família e escolha profissional: A questão espacial, temporal e o significado dos nomes. Pulsional - Revista de Psicanálise. 2005; 18(182):63-73.

27. Baptista MN. (2004). Depressão: histórico, epidemiologia, fatores de risco e modelos integrativos. In: Baptista MN. Suicídio e depressão: atualizações. Rio de Janeiro: Guanabara Koogan. 2004; p.35-49.

28. Ramos EL, Souza NVDO, Caldas, CP. Qualidade de vida do idoso trabalhador. Rev. enfermagem UERJ, Rio de Janeiro.2008; p. 507-11.

29. Chaves ÉCL, Paulino CF, Souza VHS, Mesquita AC, Carvalho FS, Nogueira DA. Qualidade de vida, sintomas depressivos e religiosidade em idosos: um estudo transversal. Texto Contexto Enfermagem. DOI http://dx.doi.org/10.1590/0104-07072014001000013.

30. Rocha FL, Melo RLP, Menezes TN. Fatores associados à síndrome metabólica em idosos do interior do Nordeste brasileiro. Revista Brasileira Geriatria Gerontologia. DOI http://dx.doi.org/10.1590/198122562016019.160046.

31. REUTER-LORENZ P, PARK DC. Human Neuroscience and the Aging Mind: A New Look at Old Problems. J Gerontol B Psychol Sci Soc Sci. DOI 10.1093/geronb/gbq035.

32. Soares E, Coelho MO, Carvalho SMR. Capacidade funcional, declínio cognitivo e depressão em idosos institucionalizados: possibilidade de relações e correlações. Revista Kairós Gerontologia. São Paulo. 2012;15(5):117-139.

33. Barreto MS, Carreira L, Marcon SS. Envelhecimento populacional e doenças crônicas: Reflexões sobre os desafios para o Sistema de Saúde Pública. Revista Kairós Gerontologia. 2015;18(1):325-339.

Schmidt MI, Duncan BB, Silva GA, Menezes AM, Monteiro CA, Barreto SM. Doenças crônicas não transmissíveis no Brasil: carga e desafios atuais. 